\title{
ECOSISTEMAS ESTRATÉGICOS Y DISPONIBILIDAD DE HÁBITAT DE LA AVIFAUNA DEL PIEDEMONTE LLANERO (COLOMBIA), COMO POSIBLE PELIGRO AVIAR ${ }^{1}$
}

\author{
Clara Inés Caro-Caro ${ }^{2}$ \\ Marco Aurelio Torres-Mora ${ }^{3}$ \\ Martha Paola Barajas-Barbosa ${ }^{4}$ \\ Recibido el 10 de diciembre de 2012 y aprobado el 3 de junio de \\ 2014, actualizado el 5 junio de 2014
}

\section{RESUMEN}

Dada la diversidad y oferta de áreas naturales o artificiales en el piedemonte Llanero y cuenca del Orinoco para la avifauna, se hace necesario llevar a cabo inspecciones y monitoreos de focos de concentración de aves como posible peligro para la aeronavegación. Para este propósito se tomó como referencia al municipio de Villavicencio (Meta, Colombia) estableciendo dos niveles diferentes de evaluación, incluyendo las áreas de aeropuertos y el Parque Ecológico Reciclante. En cada nivel identificado se realizaron visitas de inducción, ajuste de categorías de hábitats, caracterización de la cobertura y uso del suelo y la identificación de los ecosistemas estratégicos partiendo del concepto de servicios ambientales prestados, especialmente la oferta de hábitat. Las observaciones permitieron la categorización de hábitats de influencia de los niveles 1 y 2 , en ecosistemas boscosos, pastizales, ecosistemas acuáticos, agroecosistemas y la presencia de infraestructura urbana y productiva, focos importantes para aves de importancia potencial en el peligro aviario.

\section{PALABRAS CLAVE}

Biodiversidad, Orinoquia, uso y cobertura de la tierra, aeronavegabilidad, aves.

\section{STRATEGIC ECOSYSTEMS AND AVIFAUNA HABITAT AVAILABILITY IN THE PLAINS PIEDMONT (COLOMBIA) AS POSSIBLE AVIAN DANGER}

\begin{abstract}
Given the diversity and range of natural or artificial areas for avifauna in the Plains piedmont and the Orinoco river basin, it is necessary to carry out inspections and monitoring of concentration of bird outbreaks as a potential hazard to air navigation. For this purpose, the municipality of Villavicencio (Meta, Colombia) was made reference to establish two different levels of evaluation, including the areas of airports and the Reciclante Ecological Park. At each level identified induction visits, adjustment of habitat categories, coverage characterization and land use, and identification of strategic ecosystems based on the concept of environmental services, especially habitat supply were performed. The observations allowed the categorization of
\end{abstract}


level 1 and 2, habitats influence in forest ecosystems, grasslands, aquatic ecosystems, agro ecosystems and the presence of urban and productive infrastructure, central focus for birds of potential importance in aviary hazard.

\section{KEY WORDS}

Biodiversity, Orinoquia, land coverage and use, airworthiness, birds.

\section{INTRODUCCIÓN}

Las aves como integrantes significativos de los ecosistemas se usan ampliamente en estudios de biodiversidad, por ser excelentes indicadores ecológicos (Murillo, 2005) y se consideran como el constituyente de la tierra mejor conocido (Bibby, Jones \& Marsden, 1998), facilitando la evaluación y análisis de los cambios ecológicos de los sistemas; de igual forma, son una herramienta clave para la conservación de especies, de comunidades, de ecosistemas y de áreas estratégicas, para el monitoreo y para el manejo sostenible de los recursos y oferta natural de ecosistemas y paisajes. Las aves de la Orinoquia presentan asociaciones directas a los diferentes hábitats y ambientes que componen sus paisajes, dentro de ellas se encuentran un alto número de especies acuáticas o semiacuáticas asociadas a los caños, esteros y zonas inundables; especies silvícolas relacionadas con las llamadas matas de monte, bosques inundables o no inundables y especies adaptadas a las condiciones extremas y cambiantes (inundaciones, sequías y fuego) de las sabanas (McNish, 2007).

Dentro del paisaje de piedemonte de la cuenca del Orinoco, el municipio de Villavicencio constituye un sitio estratégico para las aves; con una gran diversidad debido a su ubicación y la gran oferta de hábitats, representando una zona de paso importante para especies migratorias, para la congregación especialmente de especies acuáticas y para la presencia de aves de amplia distribución y de áreas abiertas como rapaces y carroñeras. Sumado a esto, la existencia de bosques de vega, sabanas y humedales, potencializa la diversidad de la avifauna lo cual permite inferir un peligro aviar -PA- en la aeronavegación civil y militar, definido como el riesgo que supone para las aeronaves y su operación (Steenblik, 1997). Las colisiones de aviones con aves, relacionado en gran parte con el aumento de varias poblaciones de especies, y otro tipo de fauna, incrementan los problemas económicos y de seguridad (Dolbeer \& Eschenfelder, 2002). Cleary, Dolbeer \& Wright (2005) estiman que un 98\% de las colisiones con fauna se atribuye a las aves, situación que representó para Estados Unidos un costo de US\$500 millones, entre los años 1990-2004.

Las principales actividades foco de atracción para la avifauna potencialmente peligrosa para las operaciones aéreas son: existencia de lagos artificiales, actividades agrícolas y pecuarias, 
instalaciones destinadas al procesamiento de carnes y pescado, vertederos y plantas de tratamiento de basuras o residuos sólidos, rellenos sanitarios, frigoríficos, granjas pecuarias, desechos de fábricas y parques, expendios y plantas procesadoras de alimentos, que generen desperdicios comestibles para aves, ubicadas dentro de un radio de 13 kilómetros a la redonda de cualquier aeropuerto (ICAO, 2002).

Godínez (2002), destaca la importancia de identificar la diversidad de oferta como las áreas naturales o artificiales que brindan hábitat en cualquier aspecto, por ejemplo, reproducción, alimento, agua, percha, entre otros, en el área de influencia de los aeródromos y de zonas de gran importancia como los rellenos sanitarios; además, sirve como línea base para la formulación de estrategias y recomendaciones para su manejo integral en zonas de aeronavegación y de disposición de residuos sólidos. De igual forma, Blackwell, Schafer, Helon \& Linnell (2008) determinaron que las modificaciones físicas y manejo de lagos o estanques, los métodos de tratamientos de aguas artificiales y sintéticos y escenarios hidrobiológicos son efectivos para minimizar el uso de zonas de alimentación de las aves.

De acuerdo a la Organización Internacional de Aviación Civil (en su sigla en inglés ICAO), se reconocen las siguientes prácticas para el manejo del peligro de las aves en la aviación: a) evaluación de extensiones en los aeropuertos y sus alrededores como refugio para aves potencialmente peligrosas, b) acciones que permitan la disminución del número de aves, c) eliminación o prevención de algunos sitios en el aeropuerto y a sus alrededores atrayentes para las aves y de este modo evitar daños a la aviación (ICAO, 1991; Kelly \& Alla, 2006; Dolbeer, 2007). Bajo estas consideraciones, el objetivo de la presente investigación fue la evaluación de ecosistemas estratégicos y disponibilidad de hábitat de la avifauna del piedemonte Llanero como posible peligro aviar.

\section{MATERIALES Y MÉTODOS}

\section{Localización y descripción del área de estudio}

Se hizo referencia como eje de ubicación al municipio de Villavicencio, localizado al Oeste del departamento del Meta $\left(04^{\circ} 07^{\prime} \mathrm{N}, 073^{\circ} 38^{\prime} \mathrm{W}\right)$, con una extensión aproximada de 130.085 ha, con una zona urbana de alrededor de 5.427 ha (4\%), mientras que la zona rural, 124.658 ha, corresponden al $96 \%$ (Alcaldía de Villavicencio - Secretaría de Medio Ambiente, 2006). Su clima se clasifica como húmedo tropical, con una precipitación media fluctuante entre $3.484,7 \mathrm{~mm}$ a $4.484 \mathrm{~mm}$, con mayor registro de lluvias en el mes de mayo. La temperatura media es de $25^{\circ} \mathrm{C}$, con los datos más elevados para el mes de febrero; la humedad relativa (HR) varía entre el $77,2 \%$ y el $82,1 \%$, siendo los meses de junio y julio los de mayor porcentaje de HR (86\%). Se tienen medias multianuales donde se registra 
que la evaporación disminuye de $1.517 \mathrm{~mm}$ a $1.305 \mathrm{~mm} / \mathrm{año}$; la insolación real es de 1.807 horas anuales a 1.545 horas anuales de sol; y el recorrido del viento anual se reduce de $28.071 \mathrm{~km}$ a $16.013 \mathrm{~km}$ anuales, con velocidad media horaria de $1,9 \mathrm{~m} / \mathrm{s}$ a los $1,0 \mathrm{~m} / \mathrm{s}$ (Caro, Heshusius, Rodríguez, De las Casas, Rincón \& Castro, 2006; BIOTECNOS \& BIOAGRÍCOLA, 2007).

\section{Desarrollo metodológico}

La presente investigación se desarrolló en dos fases: una exploratoria y una de diagnóstico, de la siguiente manera:

\section{Fase exploratoria}

Se realizó una línea base y selección de la temática actualizada, con fuentes de orden nacional e internacional, como las propuestas por la Aeronáutica Civil y la Fuerza Aérea Colombiana, principalmente. De igual forma, se hizo una revisión cartográfica por medio de fotografías a diferentes escalas provistas por la Fuerza Aérea Colombiana, así como las imágenes generadas en el medio interactivo de Google Earth. Para una mayor delimitación del área de estudio se realizaron

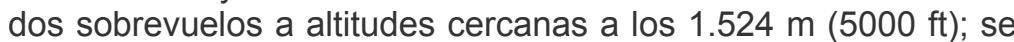
utilizó el software ArcGis-Arc View versiones 8.3 y 9.0 para la revisión y generación de la cartografía. La delimitación de la zona de estudio fue ajustada de acuerdo al manual metodológico para la caracterización y evaluación del peligro aviar presente en aeropuertos de Colombia (Amaya-Espinel \& Rico-Hernández, 2005), estableciendo dos niveles diferentes de evaluación y de análisis de metodologías apropiadas, así:

- Nivel 1. Correspondió al área comprendida por un círculo de $3 \mathrm{~km}$ de radio donde se localizaron los predios propios de cada aeropuerto y los sistemas naturales y artificiales adyacentes con gran influencia sobre el mismo.

- Nivel 2. Entendido como el área del círculo localizada entre el primer nivel y un área con un diámetro de $26 \mathrm{~km}$. Este nivel incluyó las coberturas y usos de la tierra, los ecosistemas, los agroecosistemas y la infraestructura urbana, que hacían parte del paisaje de piedemonte Llanero, adyacentes a los aeropuertos Gómez Niño y Vanguardia y al Parque Ecológico Reciclante (Figura 1). 




Figura 1. Plano de rutas de muestreo en el área de estudio. Escala 1:250.000.

\section{Fase de diagnóstico}

En cada nivel identificado se realizaron visitas de inducción, aplicando los lineamientos de bioseguridad apropiados para las condiciones logísticas. Para levantar la información primaria de hábitat, cobertura y uso del suelo, fueron ajustados los datos de georreferenciación y toponimia de las localidades objeto de estudio, mediante recorridos terrestres con una duración de tres meses; asimismo, se hicieron sobrevuelos en los meses de mayo y julio de 2009. Durante las salidas de campo se escogieron los sitios críticos de presencia de aves o posibles sitios de concentración de individuos, denominados puntos ventajosos.

Ajuste de categorías de hábitat. Una vez revisadas las condiciones ambientales del área (la heterogeneidad de hábitats y la complejidad asociada a la existencia de zonas de traslape, en el nivel 2, de las tres localidades evaluadas), se elaboró una propuesta de categorización y tipología para el área de estudio, ajustada con base en la clasificación establecida por la Aeronáutica Civil del país.

Caracterización de la cobertura y uso del suelo. El conocimiento del uso del suelo y su manejo, tanto en el nivel 1 como en el nivel 2, constituye un aspecto básico dentro del plan de manejo de fauna en los aeropuertos y en las zonas de rellenos sanitarios. La descripción de este componente se basó en la observación directa (recorridos), en el análisis de imágenes de satélite, de fotografías aéreas y de sobrevuelos, permitiendo establecer su predominancia y representatividad en esta área y en las rutas de evaluación. Se utilizó el transecto en banda, con un ancho de $20 \mathrm{~m}$ y una longitud de $100 \mathrm{~m}$, tomando al azar, un cuadrante de un $1 \mathrm{~m}^{2}$ cada $10 \mathrm{~m}$, para evaluar cobertura vegetal y especies presentes; en total se realizaron 14 transectos. La información se registró en el formato adaptado de estudios 
ecológicos rápidos -EER- (Sobrevila \& Bath, 1992), haciendo énfasis en zonas identificadas como botaderos de basura a cielo abierto, plantas de tratamiento de aguas residuales, sitios de actividades agropecuarias, económicas, industriales y otras. Se trabajó siguiendo el "Manual de uso de suelo en áreas aledañas a los aeropuertos" (Aeronáutica Civil Colombiana, 2007) y se aplicó la categorización modificada, correspondiente a las condiciones locales. El aspecto final, comparativo de referencia, se hizo con la última actualización del Plan de Ordenamiento Territorial (POT) del municipio de Villavicencio para identificar las áreas de conflicto de uso del suelo.

Identificación de ecosistemas estratégicos. Para la selección de los ecosistemas estratégicos se partió del concepto de servicios ambientales prestados, especialmente como oferta de hábitat -espejo de agua, alimento-, zonas de percha y el registro de especies de fauna en estudios precedentes. También se asignó importancia a la existencia de reservas forestales, reservas hídricas y complejos de humedales. Con relación a los sistemas productivos se incluyeron los de gran importancia a nivel local por su cobertura y efecto atrayente sobre especies de aves potencialmente peligrosas para la aeronavegación. Para evaluar los ecosistemas terrestres y ecosistemas acuáticos, los registros fueron ajustados de acuerdo a lo propuesto por Sobrevila \& Bath (1992). En las observaciones in situ, la clasificación de ecosistemas a nivel de paisaje se hizo siguiendo la propuesta de Romero, Galindo, Otero \& Armenteras (2004), para la cuenca del Orinoco colombiano. La clasificación tipo Ramsar adaptada por la Política Nacional para Humedales (Ministerio del Medio Ambiente, 2002), junto con la Resolución 0196 de 2006 de planes de manejo de humedales del Ministerio de Ambiente, Vivienda y Desarrollo Territorial -MAVDT-, fueron los referentes de los ambientes acuáticos con lo que se identificaron los bienes y servicios ambientales; esto se aplicó en algunos de los ecosistemas estratégicos más representativos de la diversidad local. De manera complementaria se describieron las comunidades vegetales dominantes en ambientes terrestres y acuáticos, tal como lo incluye el formato EER.

\section{Integración y análisis de la información}

Toda la información se trabajó en matrices; los datos de campo y la información secundaria se compilaron y se evaluaron en formatos de procesadores digitales Excel. La generación de la cartografía temática, procesamiento y análisis, se hizo con el software ArcGIS - ArcView GIS versiones 8.3 y 9.0. Se ajustó la tipología de acuerdo a las categorías establecidas en el estudio y se tuvo como referencia el modelo de datos del Instituto Geográfico Agustín Codazzi -IGAC-. Con toda la información ajustada y unificada se generaron los mapas base y temáticos. 


\section{RESULTADOS Y DISCUSIÓN}

De acuerdo a la evaluación en campo, cartográfica y al ajuste de la clasificación, los hábitats fueron agrupados bajo cinco categorías y diez tipos (Tabla 1). Los principales criterios aplicados fueron: porcentaje de área, representatividad del sistema en términos de conservación o importancia en la producción y su condición estratégica para la oferta de hábitat.

Tabla 1. Clasificación ajustada de hábitats en el área de estudio en los niveles 1 y 2

\begin{tabular}{|c|c|c|}
\hline CATEGORÍAS & TIPOS & $\begin{array}{c}\text { ÁREA } \\
\text { ESTIMADA } \\
\left(\mathrm{Km}^{2}\right)\end{array}$ \\
\hline Ecosistemas Boscosos & $\begin{array}{l}\text { Parches de bosques } \\
\text { Parches de sucesión ecológica }\end{array}$ & 1358,5 \\
\hline Pastizales & Pastizales & \\
\hline $\begin{array}{l}\text { Ecosistemas acuáticos - } \\
\text { Humedales }\end{array}$ & $\begin{array}{l}\text { Ríos } \\
\text { Humedales naturales } \\
\text { - Chucuas } \\
\text { - Pantanos } \\
\text { - Caños } \\
\text { Humedales artificiales } \\
\text { - Los canales de } \\
\text { - escurrimiento o drenaje } \\
\text { - Los sistemas piscícolas } \\
\text { - Otros }\end{array}$ & 34,000 \\
\hline Agroecosistemas & $\begin{array}{l}\text { Sistemas agrícolas } \\
\text { Sistemas avícolas y porcícolas } \\
\text { Sistemas ganaderos }\end{array}$ & 91,168 \\
\hline Infraestructura & $\begin{array}{l}\text { Infraestructura urbana y } \\
\text { productiva }\end{array}$ & 58,34 \\
\hline
\end{tabular}

\section{Caracterización y uso del suelo}

Las condiciones del suelo en el área perimetral de la pista del aeropuerto Apiay, Base Aérea CT Gómez Niño - FAC, se caracterizó por ser una pradera tipo césped jardín. Se evidenció la presencia de coberturas de bosque secundario en parches dispersos y en riberas de caños, destinadas a conservación, así como la presencia de guafales plantados (Guadua angustifolia) dispersos y matas de monte.

En la zona de influencia del aeropuerto se presentaron diversos usos del suelo relacionados con actividades productivas como cobertura herbácea (Brachiaria decumbens) para uso ganadero, presencia de piscícolas y uso de tipo turístico asociado a infraestructura recreativa: piscinas, juegos y otros. Se estimó que la cobertura dominante fue la herbácea en el nivel 1 y el uso más común la actividad ganadera, seguido del uso suburbano y del turístico. Para el caso del aeropuerto Vanguardia, en el área perimetral de la pista, el uso del suelo encontrado fue de pradera con pastos de diferente altura; en esta misma área se 
observaron actividades productivas como cultivos de arroz, cultivos de pancoger (plátano) y pastizales dedicados a la ganadería. Asimismo, se halló cobertura de bosque secundario como el bosque de vega del río Guatiquía, y el bosque de piedemonte en las quebradas La Honda, Susumuco y Vanguardia; además, vegetación plantada en cercas vivas y matas de monte. En las áreas circundantes al aeropuerto, el principal uso registrado fue el ganadero, con cobertura herbácea de Brachiaria decumbens. En el aeropuerto Apiay se evidenció actividad turística asociada a la infraestructura ya citada. Estos resultados son coincidentes con los reportados por ICAO (2002), donde se considera los usos de la tierra como contribuyentes para la presencia de fauna peligrosa para los aeropuertos, aproximadamente dentro de los $13 \mathrm{~km}$, con actividades como la ganadería, los basureros, las actividades piscícolas, la agricultura, los lagos naturales y artificiales.

Por su parte en el Parque Ecológico Reciclante -relleno sanitario de Bioagrícola-, la cobertura del suelo correspondió a la categoría "bosques y áreas seminaturales", con un área de 0,197\%, esta incluyó bosque natural fragmentado, influenciado por aprovechamiento selectivo de espacios y el establecimiento de pastos y/o agricultura de subsistencia, aunque el uso del suelo predominante es de producción forestal y de protección. Otro tipo de bosque observado fue el de galería o ripario, caracterizado por una vegetación típica de planos inundables, de vegas y de diferentes niveles de terrazas que presentan bosques de uno o dos estratos. Se desarrollan a lo largo de caños y en suelos bien a mal drenados. Su uso actual es forestal para conservación, protección y conservación, y extracción, con un $9,32 \%$ del total del área.

Además de los bosques, en esta categoría se incorporan las áreas abiertas con poca o ninguna vegetación, constituidas por playas, arenales y canales fluviales; alcanzan a cubrir el $6,82 \%$. En áreas agrícolas se registraron cultivos transitorios y cultivos permanentes. Los primeros compuestos básicamente por cultivos de arroz y de subsistencia, mientras que los segundos estuvieron representados por cultivos de importancia agroindustrial como los de palma de aceite (Eleais sp.), con un área de $6,44 \%$.

Dentro de las "áreas con pastizales" se encontraron "áreas con vegetación herbácea y/o arbustiva", incluyen pastos naturales y áreas sin uso actual -de descanso- donde domina la vegetación arbustiva; en conjunto representaron tan solo el $0,87 \%$ del total; "presencia de pastos naturales", caracterizada como la cobertura de vegetación natural o introducida compuesta por gramíneas y leguminosas en la que se observa algún tipo de manejo agronómico. El uso predominante identificado fue la ganadería extensiva, con presencia de pastos y arbustales de origen natural y/o antrópica, materia prima para esta actividad en la zona de estudio.

Otros tipos hallados en las "áreas con pastizales", fueron los "pastos manejados", conformados por plantas herbáceas 
cultivadas con períodos vegetativos anuales y con mayores rendimientos y valores nutritivos dentro de la categoría de tierras agrícolas; son materia prima para la ganadería extensiva; y los "pastos introducidos" integrados por especies vegetales herbáceas foráneas, importantes para la obtención de altos rendimientos y valores nutritivos en la ganadería semi-intensiva. En general, los usos del suelo más comunes en toda la zona de estudio se relacionan con el sector agropecuario, la infraestructura urbana y los ecosistemas naturales acuáticos y terrestres, siendo los campos agrícolas hábitats y fuente de alimento de las aves durante la época de invierno (Perkins, Whittingham, Bradbury, Wilson, Morris \& Barnett, 2000).

\section{Identificación de Ecosistemas estratégicos y disponibilidad de hábitat para aves}

Los sistemas de bosque que forman parte de los niveles 1 y 2 de los tres sitios estudiados correspondieron a bosques secundarios, que presentan estructuras semiabiertas debido a la extracción selectiva de recursos maderables; en algunos de ellos se registró la acción directa del pastoreo y del paso de ganado y eventualmente de agricultura de subsistencia. Estos ecosistemas registraron diversos estados sucesionales; en la reserva Buenavista y en el nivel 1 del Parque Ecológico Reciclante se evidenció este proceso ecológico, representado en praderas con pequeñas manchas de rastrojos. De acuerdo a Romero et al. (2004), este tipo de bosques posee un sistema de comunidades ecológicas y biodiversidad complejas, con un alto nivel de estratificación y una gran diversidad de especies, en relación con la diversidad florística explicada en la evolución biogeográfica y la historia climática de la región. Esta complejidad estructural del hábitat es uno de los principales factores ecológicos causantes de la alta diversidad de especies en las comunidades de aves tropicales (Marra \& Remsen, 1997).

Dentro de los remanentes del bosque andino se observó la presencia de epífitas, identificando hábitats como los parches de bosques y los parches de sucesión ecológica presentes en todos los sitios estudiados, pero con mayor cobertura en área en la zona del Parque Ecológico Reciclante.

La mezcla de actividades agropecuarias, de expansión urbana y turística, sin aplicar el ordenamiento territorial en áreas urbanas y rurales - niveles 1 y 2 , propicia la ampliación de las zonas de "pradera" y de pastizales, con especies introducidas como la $B$. decumbes y oferta nuevos hábitats para especies de avifauna propias de áreas abiertas y para especies de aves oportunistas y para otros taxa. Esta cobertura tiene un gran porcentaje dentro del área total de los tres sitios evaluados, con una mayor extensión en el nivel 1, en el área del aeropuerto Gómez Niño y en el área del Parque Ecológico Reciclante, y en menor porcentaje en el aeropuerto Vanguardia, donde son reemplazados por cultivos de arroz y viveros. En el nivel 2, dominan en el Parque Ecológico Reciclante. 
Los ecosistemas acuáticos presentes dentro de los ecosistemas estratégicos fueron clasificados en dos tipos, ríos y humedales. En el primer tipo fueron identificadas tres cuencas relacionadas con la oferta de hábitats potenciales para fauna importante en el peligro aviar.

Río Guatiquía: en el municipio de Villavicencio recorre las veredas Quebrada Honda, Lourdes, Puente Abadía, San José, San Cristóbal, Santa Helena, Santa Teresa y Santa María Baja, entre otras. Su oferta de hábitat se caracterizó por la captación de aguas con vertimientos de sólidos y líquidos (vía tributarios), junto con los vertimientos directos a su cauce; los productos ambientales que brinda, como agua y material para construcción, lo convierten en un foco para la fauna incidente en el peligro aviar, de manera particular para aves carroñeras.

Río Ocoa: la cuenca se localiza en la parte central del municipio de Villavicencio, en dirección occidente-oriente, recibe cargas tributarias de cerca de 30 cuerpos de agua entre los que se encuentran los caños Maizaro, Buque, Colepato, La Virgen, Pescado y Corcovado. Su trayecto se extiende a lo largo de 68 $\mathrm{km}$ aproximadamente y tiene un área estimada de 27.665 ha. Debido a las altas cargas contaminantes de la mayor parte de sus tributarios, a la disposición directa de residuos sólidos y líquidos en su cauce, a las distintas actividades productivas que soporta de forma directa e indirecta (porcicultura, turismo, desarrollo industrial, entre otras), constituye un sitio crítico para la oferta de hábitats a fauna asociada con peligro aviar.

Río Guayuriba: desde su parte alta recibe numerosas descargas de centros poblados, de actividades agropecuarias y de actividades turísticas. Su influencia principal se relaciona con la oferta de hábitat, en el nivel 2, para las tres localidades objeto de estudio. Con la presencia de aves con potencial peligro aviar se hace necesario su valoración. De acuerdo a Blackwell, DeVault \& Fernández-Juricic (2009), actividades mínimas y menos importantes son las siguientes: 1) Análisis de eventos y circunstancias que requieren de valoración, 2) Identificación de especies observadas, número, ubicación, movimientos y ocurrencia durante las estaciones, 3) Identificación y localización de futuras y nuevas zonas atractivas para la fauna, 4) Descripción de la fauna peligrosa para las operaciones aéreas y 5) Acciones y recomendaciones para la reducción de fauna peligrosa para las operaciones aéreas.

Por su parte, los humedales tienen una alta representatividad dentro del paisaje de piedemonte donde se localiza la zona de estudio y se ubican tanto el nivel 1 como en el nivel 2.

- Humedales naturales. Integrado por chucuas, pantanos y caños. Cabe destacar que en lo observado, los pantanos tenían uso como botaderos a cielo abierto o para la disposición final de material de construcción, lo que conlleva a una acumulación de desechos y por ende al mantenimiento de una fuente de recursos alimenticios para la avifauna. 
- Humedales artificiales. En esta categoría se agrupan diversos tipos de sistemas: los primeros representados por canales de escurrimiento o drenajes, asociados principalmente a las pistas de aterrizaje y colindantes con el nivel 1 , son particularmente importantes en la oferta de hábitat a especies de aves acuáticas en la época seca y a otros grupos de fauna durante todo el año.

Los sistemas piscícolas, como segundo tipo registrado, tradicionalmente presentan conflicto con las aves acuáticas, puesto que su oferta de alimento genera un hábitat deseable para una diversidad de especies. A pesar de los diferentes métodos de control que se aplican (mallas, sonidos, cacería) la abundancia de alimento mantiene a varios gremios tróficos.

Como tercer tipo identificado, los cultivos de arroz ofrecen un hábitat acuático a diversa fauna, especialmente a las aves acuáticas y aves migratorias; estos cultivos fueron localizados principalmente en los niveles 1 y 2 de los aeropuertos Vanguardia y Gómez Niño.

Finalmente en el tipo "otros", se agruparon las piscinas y construcciones de almacenamiento de agua para consumo humano, para abastecimiento de ganadería, de agricultura u otras actividades.

Las tres zonas objeto de estudio se localizan, en su mayor parte, en lo que corresponde al municipio de Villavicencio. Lo que se delimita como niveles 2, área de diámetro de $26 \mathrm{~km}$, en cada caso, tiene traslape, tal como se puede observar en la Figura 2. En el mapa se contrasta con color naranja aquellos usos contradictorios con el uso aprobado por el POT (Acuerdo 021), especificando en las convenciones el tipo de uso y su relación con los hábitats existentes. De ese modo, se tienen usos en la reserva Buenavista y en la reserva Vanguardia, sobre la ribera de cursos de agua (como en el río Ocoa) que no corresponden a lo estipulado en el ordenamiento ambiental territorial.

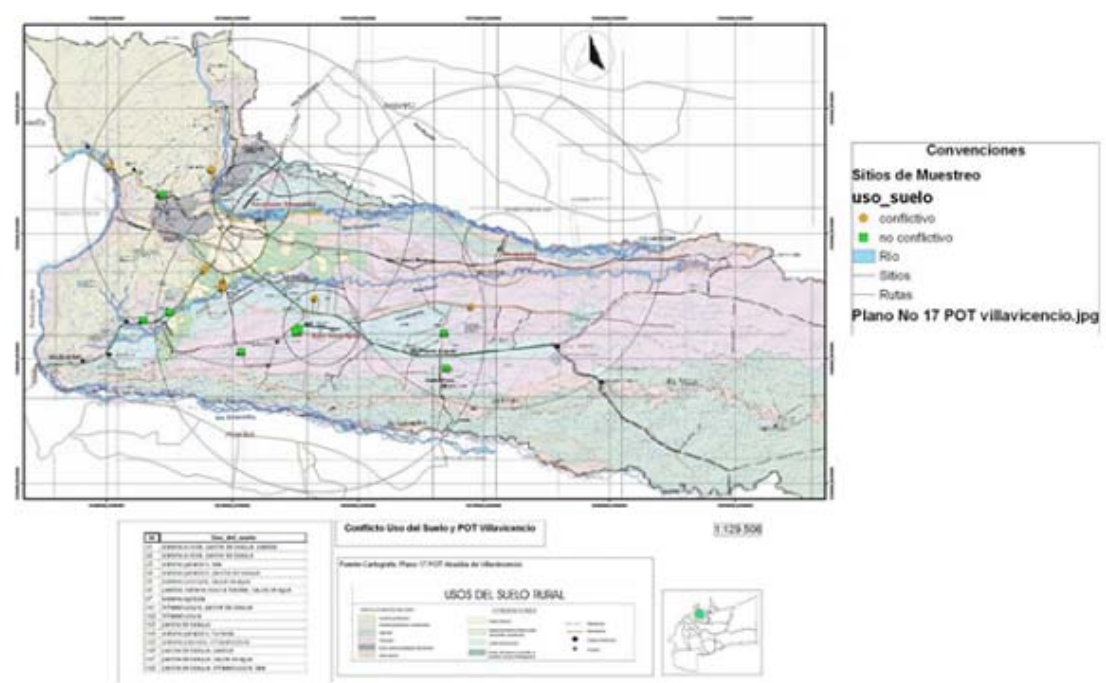

Figura 2. Conflicto de uso del suelo vs POT Villavicencio. Mapa adaptado. Escala $1: 130.000$ 
La mayor área en traslape la comparten los dos aeropuertos, con mayor área en infraestructura (Villavicencio) y en bosques (zonas de reserva), seguida de la zona de traslape aeropuerto Gómez Niño-Parque Ecológico Reciclante, principalmente en lo relacionado con actividades agropecuarias y presencia de parches de bosque.

\section{CONCLUSIONES}

Los ecosistemas estratégicos más importantes en la oferta de hábitat para la avifauna asociada al peligro aviar son los ríos Guatiquía, Ocoa y Guayuriba; los agroecosistemas de tipo pecuario y avícola; los humedales naturales y artificiales. El aeropuerto Vanguardia presenta el mayor efecto de impacto en peligro aviar debido a la gran oferta de hábitat, en términos de área y de diversidad asociado a su ubicación y a la acción deficiente de las autoridades ambientales que posibilitan conflictos de uso del suelo y focos numerosos para aves carroñeras y de potencial para el peligro aviario. En contraste, el Parque Ecológico Reciclante -relleno sanitario- posee la oferta más baja en área de infraestructura y bosques, y una gran oferta asociada al hábitat de pastizal, con presencia de sistemas lóticos como los ríos Guatiquía y Ocoa, focos significativos para aves de importancia potencial en el peligro aviario.

Finalmente, el manejo del peligro aviar en el área de estudio necesita un trabajo integrado de control sobre los focos atrayentes de fauna silvestre potencialmente peligrosa para la aeronavegación, mediante acciones sinérgicas de los responsables del manejo en el nivel uno (administradores de aeropuertos y del relleno sanitario) y de las autoridades ambientales locales (Alcaldías, CAR) como responsables del manejo y administración de los recursos naturales en el nivel 2.

\section{AGRADECIMIENTOS}

Los autores agradecen a la Aeronáutica Civil, Fuerza Aérea Colombiana y a la Universidad de los Llanos (Convenio Interadministrativo No. 8000127-OH-2008/Unillanos 071 suscrito entre la Unidad Administrativa Especial de la Aeronáutica Civil, Fuerza Aérea Colombiana - Comando Aéreo de Combate No. 2 y la Universidad de los Llanos), y a todas aquellas personas que en desempeño de sus funciones institucionales 0 en acompañamiento al trabajo de campo y recorridos apoyaron el correcto desarrollo de este proyecto. 


\section{REFERENCIAS}

- Aeronáutica Civil Colombiana. (2007). El uso de suelos en áreas aledañas a los aeropuertos. Recuperado de http://portal.aerocivil.gov.co/portal/pls/portal/!PORTAL.wwpo b_page.show?_docname $=751210$. PDF

- Alcaldía de Villavicencio - Secretaría de Medio Ambiente. (2006). Sistema de Gestión Ambiental "SIGAM" Municipio de Villavicencio. Perfil Ambiental y Plan de Acción. Informe Final. Villavicencio: CORPOLLANOS.

- Amaya-Espinel, J. D. \& Rico-Hernández, G. (2005). Caracterización y evaluación del peligro aviario presente en siete aeropuertos de Colombia. Informe Final y Plan de Manejo, Aeronáutica Civil de Colombia - Unión Temporal UTAR Manejo Peligro Aviario. Contrato de consultoría No. 4000220, Bogotá.

- Bibby, C., Jones, M. \& Marsden, S. (1998). Expedition Field Techniques Bird Surveys. London: Royal Geographical Society Institute of British Geographers.

- BIOTECNOS \& BIOAGRÍCOLA. (2007). Informe Técnico de los componentes abióticos y bióticos del estudio de impacto ambiental del Parque Ecológico Reciclante. Informe técnico de avances. Contrato 2.705-68 de 2005, Villavicencio.

- Blackwell, B. F., DeVault, T. L. \& Fernández-Juricic, E. (2009). Wildlife collisions with aircraft: A missing component of land-use planning for airport. Landscape and Urban Planning, 93, 1-9.

- Blackwell, B. F., Schafer, L. M., Helon, D. A. \& Linnell, M. A. (2008). Bird use ofstormwater management ponds: decreasing avian attractans on airport. Landscape Urban Plan, 86, 162-170.

- Caro, C. I., Heshusius, M., Rodríguez, C., De las Casas, J. C., Rincón, R. \& Castro, F. (2006). Estudio de Fauna del Municipio de Villavicencio. Informe Técnico Final. Convenio 021, Alcaldía de Villavicencio, Secretaría de Medio Ambiente, Universidad de los Llanos, Villavicencio.

- Cleary, E. C., Dolbeer, R. A. \& Wright, S. E. (2005). Wildlife Strikes to civil aircraft in the United Sates, 1990-2004. U.S. Obtenido de Departament of Transportation, Federal Aviation Administration: http://wildlife-mitigation.tc.faa.gov

- Dolbeer, R. A. (2007). Managing the risks of bird strikes. Int Airport Rev, 2, 61-64.

- Dolbeer, R. A. \& Eschenfelder, P. (2002). Population increases of large birds, airworthiness standards and high-speed flight: a precacrious combination. En Proceedings of the 55th International Air Safety Seminar (pp. 273-281). Dublin, Ireland: Flight Safety Foundation, Alexandria, VA, USA.

- Godínez, E. (2002). Programa Nacional de Limitación de la fauna silvestre en Aeropuertos de Panamá. Proyecto PAN/99/801, Ministerio de Cooperación Técnica de la OACl en Panamá.

- International Civil Avaitaion Organization -ICAO-. (1991). Bird Control and reuction. Airport Services Manual. Doc 9137-AN/898, part 3.

- (2002). Land use and environmental control. Airport Planning Manual. Doc 9184-AN/902, part 3.

- Kelly, T. C. \& Allan, J. (2006). Ecological effects of aviation. En Davenport, J. \& Davenport, J. L. (Eds.). The Ecology of Transportation: Managing Mobility for the Environment (pp. 5-24). The Netherlands: Springer. 
- Marra, P. P. \& Remsen, J. V. (1997). Insights into the maintenance of high species diversity in the neotropics: habitat selection and foraging behavior in understory birds of tropical and temperate forests. Ornitological Monographs, 48, 445-483.

- McNish, T. 2007. Las aves de los Llanos Orientales de Colombia. Bogotá: M y B. 302 pp

- Ministerio del Medio Ambiente. (2002). Política Nacional para Humedales Interiores de Colombia. Estrategia para su conservación y uso sostenible. Dirección General de Ecosistemas - Ministerio del Medio Ambiente, Bogotá.

- Murillo, J. I. (2005). Evaluación de la distribución y estado actual de los registros ornitológicos de los Llanos Orientales de Colombia. Trabajo de Grado para optar el título de Biología con Énfasis en Ecología, Universidad de Nariño, Pasto.

- Perkins, A. J., Whittingham, M. J., Bradbury, R. B., Wilson, J. D., Morris, A. J. \& Barnett, P. R. (2000). Habitat characteristics affecting use of lowland agricultural grassland by birds in winter. Biology conervation, 95, 279-294.

- Romero, M., Galindo, G., Otero, J. \& Armenteras, D. (2004). Ecosistemas de la Cuenca del Orinoco Colombiano. Bogotá, Colombia: Investigaciones de Recursos Biológicos Alexander von Humboldt.

- Sobrevila, C. \& Bath, P. (1992). Evaluación Ecológica Rápida. Un Manual para usuarios de América Latina y el Caribe. TNC.

- Steenblik, J. W. (1997). Wildlife hazards to aircraft. Air Line Pilot, 66, 30-33.

1. Convenio Interadministrativo No. $8000127-\mathrm{OH}-2008 /$ Unillanos 071 suscrito entre la Unidad Administrativa Especial de la Aeronáutica Civil, Fuerza Aérea Colombiana - Comando Aéreo de Combate No. 2 y la Universidad de los Llanos para el desarrollo del proyecto: "Incidencia de aves y prueba de métodos para el control de peligro aviario y proliferación de aves de carroña en la Base Aérea de Apiay, Aeropuerto Vanguardia y Parque Ecológico Reciclante".

2. Biólogo, Universidad Nacional de Colombia. Magíster en Ecología. Docente Investigador. Grupo de Investigación en Gestión Ambiental Sostenible -GIGASclarainescaro@unillanos.edu.co Grupo de Investigación en Gestión Ambiental Sostenible GIGAS-, Facultad de Ciencias Básicas e Ingeniería. Universidad de los Llanos km 12 vía Puerto López, A.A. 110, Villavicencio, Meta, Colombia.

3. Biólogo, Universidad Nacional de Colombia. Ph.D en Energía y Tecnologías del Medio Ambiente para el Desarrollo. Docente investigador. Grupo de Investigación en Gestión Ambiental Sostenible-GIGAS-. marcotorres@unillanos.edu.co

4. Biólogo, Universidad Javeriana. Grupo de Investigación en Gestión Ambiental Sostenible -GIGASpaolabarajas@gmail.com 
Para citar este artículo: Caro-Caro, C.I., Torres-Mora, M.A, Barajas-Barbosa, M.P. (2014). Ecosistemas estratégicos y disponibilidad de hábitat de la avifauna del piedemonte llanero (Colombia), como posible peligro aviar. Revista Luna Azul, 39, 25-39. Recuperado de http://lunazul.ucaldas.edu.co/index.php?option=content\&tas k=view\&id=946 